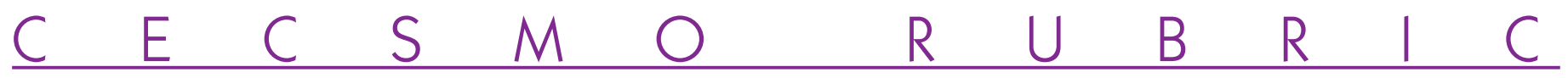

\title{
Evaluation of the effectiveness of indirect bonding of orthodontic attachments with RMGIC Resin Modified Glass-lonomer Cement
}

\author{
Amaryllis WURTH \\ Supervised by Jean-Pal FORESTIER, Marius DUMITRACHE \\ Department of Orthodontics University of Paris, Diderot-Paris 7, Paris
}

\begin{abstract}
This article reports our study, carried out as a CECSMO assignment, assessing the effectiveness of resin modified glass ionomer cement as a bonding agent on enamel over a two year period and a consideration of the relative influence of two brands of attachments, Orthos and Damon. In our study group we had an overall rate of bonding rupture of $11.6 \% .17 \%$ of direct and $33 \%$ of the indirect bondings loosened in the first trimester. Globally, the direct bonding had a higher survival rate, which was, after one year, 95.2\% for the direct bonding and 90.7\% for the indirect. The survival rate of attachments bonded to second molars was the lowest for all the teeth in the arch with Damon Indirect recorded at .889 after a year and Orthos Indirect .524 after a year. We categorized our results in relation to the characteristics of our sample (its size, its heterogeneity, and the duration of the observation period) and in relation to the characteristics of the attachments (their size and the surface and type of their bases) and in relation to the protocols (of indirect bonding, of preparation of enamel surface, of photopolymerization, of placement of the first arch, of bracket size, of ligature placement, and type of mechano-therapy used).
\end{abstract}

Our study confirmed the hypothesis that direct or indirect bonding with Fuji Ortho $L C^{\otimes}$ functions effectively in the daily practice of orthodontics. Our rates of accidental attachment debonding and our rates of bonding survival were acceptable except in Orthos indirect bonding of mandibular teeth. We found that under the conditions of our study direct bonding functioned slightly better than indirect bonding. Practitioners need to know and understand certain parameters influencing the use of resin modified glass-ionomer cement for bonding orthodontic attachments in order to optimize its success notable with regard to the choice of attachments with respect to bonding material or the effect of overbite on retention of attachments. Furthermore, the multiplicity of interfaces and of materials utilized in indirect bonding weakened bond strength under the conditions and protocols of our study.

Address for correspondence: A. WURTH, 23 rue Hofacker, 67205 Oberhausbergen, Amaryllis.wurth@laposte.net

\section{KEYWORDS}

Resin modified glass-ionomer cement (RMGIC)

Bonding

Enamel. 


\section{1 - INTRODUCTION}

Bonding of attachments is an everyday activity in our practices; it is a key element in our therapeutic efforts, one that contributes to the success of our treatments and which plays an important role in the way we organize our daily work. The inconvenient aspects of bonding with composites include the difficulty of controlling saliva and air humidity and the pollution and contamination that can cause unsightly discoloration and even decalcification of enamel surfaces especially with patients whose oral hygiene is poor and sometimes iatrogenically from the use of rotary instruments in the debonding process.

Considering the advantages and disadvantages of bonding with resin modified glass-ionomer cement we concluded that an evaluation of how effective it is in daily practice would be a worthwhile endeavor. In addition,

\section{2 - METHODS AND MATERIALS}

\section{2 - 1 - Our study design}

This was a transverse monocentric study on two parallel treatment groups. The data collection was entirely retrospective.

\section{2 - 2 - Our sample}

All the patients in our sample were treated by Dr. F. Roussarie.

\section{2 - 2 - 1 - Criteria for selection of patients in sample}

All of the patients, who were treated, as already indicated, by the same practitioner, were teen-agers whose dentitions had already matured into at least the adolescent phase. Some had with the objective of improving our indirect bonding technique and limiting the need for rebonding compared to direct methods, we were interested in learning if RMGIC could be used with an indirect protocol.

In a preliminary study we tried to get answers to two questions:

- Is there a difference in the rate that attachments become detached between direct and indirect bonding techniques?

- What effect do type of attachment and type of tooth have on the rate of detachment in the indirect technique?

This is a primary phase of our study designed to serve as a point of departure for a future study comparing the lengths of survival attachments bonded RMGIC with the survival of attachments bonded with composites.

oral habits, some did not. But all had undergone orthodontic treatment, some with, some without extractions, of at least two years in duration. All the teeth we bonded in the study were fully erupted and in occlusion.

\section{2 - 2 - 2 - Criteria for excluding patients from the sample}

We excluded from our sample all patients who had excessive salivary flow, who were hyper-active, who had metallic or ceramic crowns or amalgam or ceramic fillings on the buccal bonding surface of their teeth, had teeth with orthodontic bands, had temporary teeth still in full-banded orthodontic treatment or had already 
experienced detachment of bonded brackets.

\section{2 - 2 - 3 - Data collected for each patient}

For each patient we filled out a chart, in table form, that included the initials of the patient, age, sex, any special behavioral patterns including oral habits. Later, as the study proceeded, we added data relevant on each bonded tooth: the date patient's participation began the date of first bond failure, dates of rebonding, state of rebonded teeth, date of termination of participation and the condition of the bonded attachments at that time.

\section{2 - 2 - 4 - Characteristics of our sample}

Our sample consisted of 120 teenagers, with a slight preponderance of females, with an average age of 13.2 years.

\begin{tabular}{|c|c|c|}
\hline & & Test $\chi^{2}$ \\
\hline \multicolumn{3}{|l|}{ Number of subjects } \\
\hline $\begin{array}{l}\text { Total } \\
\text { Girls } \\
\text { Boys }\end{array}$ & $\begin{array}{l}116 \\
67 \text { (58\%) } \\
49(42 \%)\end{array}$ & $\begin{array}{c}\mathrm{p}=\underset{\text { (non- }}{0,0026} \\
\text { homogenous } \\
\text { groups) }\end{array}$ \\
\hline \multicolumn{3}{|l|}{ Distribution of subjects by age } \\
\hline $\begin{array}{l}\text { Under } 12 \text { years } \\
\text { From } 12 \text { to } 14 \text { years } \\
\text { From } 14 \text { to } 16 \text { years } \\
\text { From } 16 \text { to } 18 \text { years }\end{array}$ & $\begin{array}{cc}64 & (55 \%) \\
36 & (31 \%) \\
8 & (7 \%)\end{array}$ & $\begin{array}{c}\mathrm{p}<2,2 \times 10^{-16} \\
\text { (non- } \\
\text { homogenous } \\
\text { groups) }\end{array}$ \\
\hline \multicolumn{3}{|c|}{ Distribution according to type of bonding } \\
\hline $\begin{array}{l}\text { Total number of attachments } \\
\text { Number of attachments } \\
\text { bonded directly: } \\
\text { Orthos direct technique (OD) } \\
\text { Number of attachments } \\
\text { bonded indirectly: } \\
\text { Orthos indirect technique (OI) } \\
\text { Damon indirect technique (DI) }\end{array}$ & $\begin{array}{l}372(20 \%) \\
650(35 \%)\end{array}$ & $\begin{array}{c}\mathrm{p}=5,3 \times 10^{-9} \\
\text { (non- } \\
\text { homogenous } \\
\text { groups) }\end{array}$ \\
\hline
\end{tabular}

Table 1

Distribution of the sample according to sex, age, and type of bonding
We bonded at total of 1862 Orthos (ORMODENT ${ }^{\circledR}$ ) OR Damon (ORMOD$\mathrm{ONT}^{\circledR}$ ) using direct and indirect methods on four types of teeth, incisors, canines, premolars, and molars. We subdivided the molar group into two sectors, first molars and second molars.

These characteristics are displayed in tables 1 and 2.

\section{2 - 3 - Evaluating bonding Procedures}

\section{2 - 3 - 1 - Types of attachments used}

Orthodontic treatment was accomplished with the aid of metal Orthos (ORMODENT ${ }^{\mathrm{TM}}$ ) or of first generation metallic Damon ORMODENTTM) attachments for all the teeth except the molars and for Orthos (ORMODENT ${ }^{\mathrm{TM}}$ ) or first generation metallic Damon

\begin{tabular}{|c|c|c|c|c|}
\hline $\begin{array}{l}\text { Distribution } \\
\text { of attachments }\end{array}$ & Total & Max & Mand & $\begin{array}{c}\text { Test } \chi^{2} \\
\text { par groupe }\end{array}$ \\
\hline \multicolumn{5}{|c|}{ Orthos in indirect technique (OI) } \\
\hline $\begin{array}{l}\text { Molars } \\
\text { Premolars } \\
\text { Canines } \\
\text { Incisors }\end{array}$ & $\begin{array}{c}102 \\
108 \\
51 \\
111\end{array}$ & $\begin{array}{l}55 \\
53 \\
27 \\
60\end{array}$ & $\begin{array}{l}47 \\
55 \\
24 \\
51\end{array}$ & $\begin{array}{l}p=0,8729 \\
\text { (homogenous } \\
\text { groups) }\end{array}$ \\
\hline \multicolumn{5}{|c|}{ Damon in indirect technique (DI) } \\
\hline $\begin{array}{l}\text { Molars } \\
\text { Premolars } \\
\text { Canines } \\
\text { Incisors }\end{array}$ & $\begin{array}{c}161 \\
202 \\
90 \\
197\end{array}$ & $\begin{array}{c}93 \\
128 \\
53 \\
120\end{array}$ & $\begin{array}{l}68 \\
74 \\
37 \\
77\end{array}$ & $\begin{array}{l}\mathrm{p}=0,7271 \\
\text { (homogenous } \\
\text { groups) }\end{array}$ \\
\hline \multicolumn{5}{|c|}{ Orthos in direct technique (OD) } \\
\hline $\begin{array}{l}\text { Molars } \\
\text { Premolars } \\
\text { Canines } \\
\text { Incisors }\end{array}$ & $\begin{array}{l}113 \\
249 \\
147 \\
331\end{array}$ & $\begin{array}{c}32 \\
152 \\
81 \\
201\end{array}$ & $\begin{array}{c}81 \\
97 \\
66 \\
130\end{array}$ & $\begin{array}{l}\mathrm{p}=8,08 \cdot 10^{-9} \\
\text { (homogenous } \\
\text { groups) }\end{array}$ \\
\hline Total & 1862 & 1055 & 807 & \\
\hline
\end{tabular}

Table 2

Distribution of the sample according to type of bonding and type of tooth. 
ORMODENT'M ) tubes on the molars. The bracket slot was $022 \times 028$.

\section{2 - 3 - 2 - Bonding material used in the study}

We evaluated GC Fuji Ortho LC (GC Corp. ${ }^{\mathrm{TM}}$ ) cement, which is photopolymerizable and prepared in the form of fixed dosage sealed capsules. We prepared tooth surfaces for bonding with a commercial $10 \%$ solution of acrylic acid marketed as GC Fuji Ortho LC Conditioner (GC Corp. ${ }^{\mathrm{TM}}$ ). We kept all the products used in the study under refrigeration.

\section{2 - 3 - 3 - Direct bonding method used in the study} faces.

- Cleaning of the bonding sur-

- Isolation of the operative field.

- Enamel preparation: application of the conditioner (20seconds) followed by 20 second rinsing and a short drying stage of fewer than 5 seconds.

- Bonding of the attachments: activation of the capsule, mixing in the amalgamator for 10 seconds, placing the RMGIC on the base of the attachment, placing the attachment on the tooth where it was held under light pressure, and removal of excess bonding agent with an explorer or a scaler.

- Photopolymerization of the bonding agent for 10 seconds with a LED DEMETRON ${ }^{\top M}$ lamp whose power was $1500 \mathrm{~mW} / \mathrm{mm}^{2}$.

- Removal of any remaining excess RMGIC with a bond material removing bur

- Immediate incorporation of the attachments in appliance by ligating a memory alloy orthodontic wire, usually .014 diameter, to them.

\section{2 - 3 - 4 - Indirect bonding method used in the study}

- Cleaning of the bonding surfaces.

- Try-on of splints and cleaning.

- Isolation of operative field.

- Enamel preparation: application of the conditioner (20seconds) followed by 20 second rinsing and a short drying stage of fewer than 5 seconds.

- Bonding of the attachments: activation of the capsule, mixing in the amalgamator for 10 seconds, placing the RMGIC on the base of the attachment, placement of the splint.

- Two stage photopolymerization with a LED Very Light EVOLUTION ${ }^{T M}$ lamp for 5 seconds with the splint in place for the incisors and for $10 \mathrm{sec}-$ onds with the splint in place for the other teeth, and then for an additional 10 seconds with the splints removed.

- Removal of excess RMGIC with bond material removing bur and dental floss.

- Immediate incorporation of the attachments in appliance by ligating a memory alloy orthodontic wire, usually .014 diameter, to them.

\section{2 - 3 - 5 - Protocol for the labora- tory preparation of a transfer splint}

\section{- Material}

- Pre-bonding material: Transbond XT (3M UNITEK ${ }^{\mathrm{TM}}$ ) composite stored in a refrigerator.

- Photopolymerization material: an Optilux $501 \mathrm{KERR}^{\mathrm{TM}}$ halogen lamp, $540 \mathrm{~mW} / \mathrm{cm}^{2}$ power.

- Material for pouring the impression: Quick Dye (BISICO ${ }^{\mathrm{TM}}$ ) and Rigidur (BISICO TM). 
- Thermoformed acrylic plates: Mouthguard $1 \mathrm{~mm}$ Dentsply (GACSOFTM) and Ideal Clear, Ideal Smile (GAC-SOF).

- Separating solution: DSL 16 Multi-purpose Silicine Lubricant (DENTRONIXTM) and Blue Freeze $-50^{\circ} \mathrm{C}$ (STERIBLUETM).

\section{- Method}

- Taking and cleaning the impression;

- Pouring the impression in Quick Dye and its base in Rigidur;

- Placing of Transbond XT composite and its photopolymerization for 10 seconds;

- Construction of the flexible internal splint to be covered with an external rigid splint;

\section{3 - RESULTS}

We shall present our results in three sectors according to the duration of survival, the rate of accidental detachment, and the survival rate.

\section{3 - 1 - Duration of survival}

The global duration rate of attachment retention in our study is 795 days, which is approximately two years and two months. The average duration of retention of attachments of the Orthos Direct group is the longest of the three assessed, 892 days. The other two had average duration rates that were about the same, 719 days for the Orthos indirect group and 774 day for the Damon indirect group.

\section{3 - 2 - Rate of debonding}

The over-all rate of bonding for the three groups was $11.6 \%$.

The rate of debonding for attachments in the Orthos Indirect group
- Setting the splints on the model.

\section{2 - 3 - 6 - Analysis method}

The criterion for evaluation will be the survival of the attachment: for each tooth at the latest 2 years after inclusion, no matter what the cause. The software programs will be Excel for the data base and $\mathrm{R}$ for the statistical analysis. The survival curves will be constructed according to the Kaplan and Meier method. The comparison of the lengths of life of the attachments bonded with Fuji Ortho LC will be obtained and compared with the two survival curves by Peto's test of Log Rank (1972).

was $21 \%, 78$ attachments debonded out of the 372 that were bonded. For the Damon indirect group the rate was $10.5 \%, 68$ debonded out of the 650 bonded. And for the Orthos direct group the rate was $8.5 \%, 71$ detached out of 840 bonded. For the indirectly bonded attachments, $33 \%$ of the debondings occurred after 3 months and $50 \%$ within 6 months. These percentages are lower in the group of directly bonded attachments, where the figures were $17 \%$ of debondings taking place in the first trimester and $37 \%$ occurring within the first six months.

\section{3 - 3 - Survival rate}

\section{3 - 3 - 1 - Survival rate according to the type of bonding}

Globally, the attachments bonded directly had a statistically significant ( $p=.0149$ ) better survival rate. On the 
average, after 6 months, $96.9 \%$ of he directly bonded attachments and $92.7 \%$ of the indirectly bonded attachments were still in place. After a year $95.2 \%$ of the directly bonded attachments and $90.7 \%$ of those bonded indirectly remained in position. After two years $92.2 \%$ of the directly bonded attachments and $88 \%$ of those bonded indirectly had not become detached.

\section{3 - 3 - 2 - Survival rate as a function of the dental sector (fig. 2)}

The attachments affixed to the second molars were the ones that detached at



Figure 1

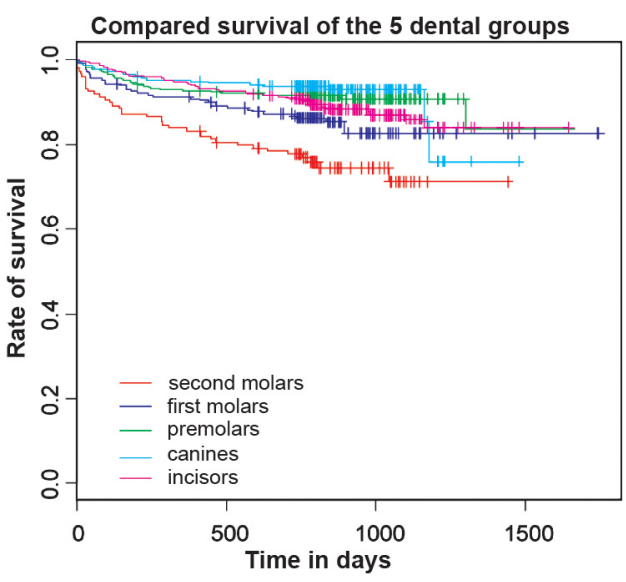

Figure 2 the highest rate: $87.2 \%$ were in place after six months, 83.8\% after one year, and $77.6 \%$ after two years. That the locale of these teeth influenced the length of time their attachments stayed in place is shown by the survival rate being statistically different for the five groups $\left(p=1.82 \times 10^{-08}\right)$.

\section{3 - 3 - 3 - Survival rate as a function of the dental sector of the arch and the type of bonding}

The attachments bonded to the second molars (fig. 3) and the first molars (fig. 4) were retained best in the Orthos direct group, a little less well in the Damon indirect group, and the least satisfactorily in the Orthos indirect group over a two-year treatment period. The attachments of the three groups had a better survival rate on maxillary molars than they did on mandibular molars.

Indirect bonding of Damon attachments registered a performance similar to that of Orthos attachments for the premolar sector (fig.5). Our results indicated that Orthos attachments should not be bonded indirectly in the mandible because for the first molars their survival rate there was only .764

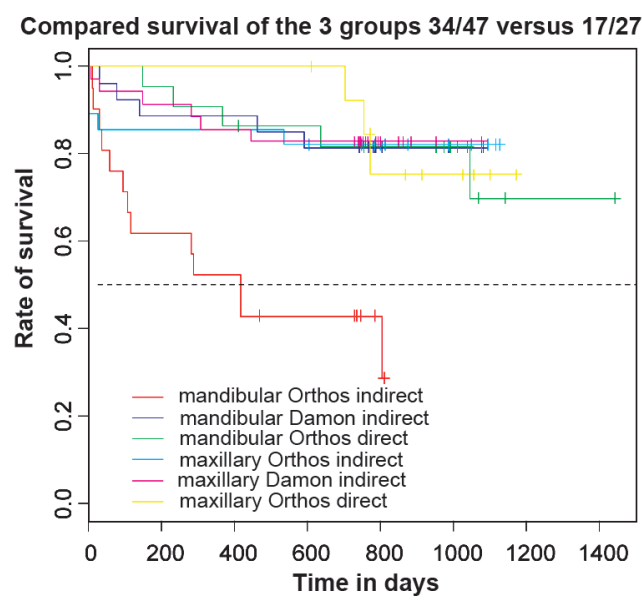

Figure 3 


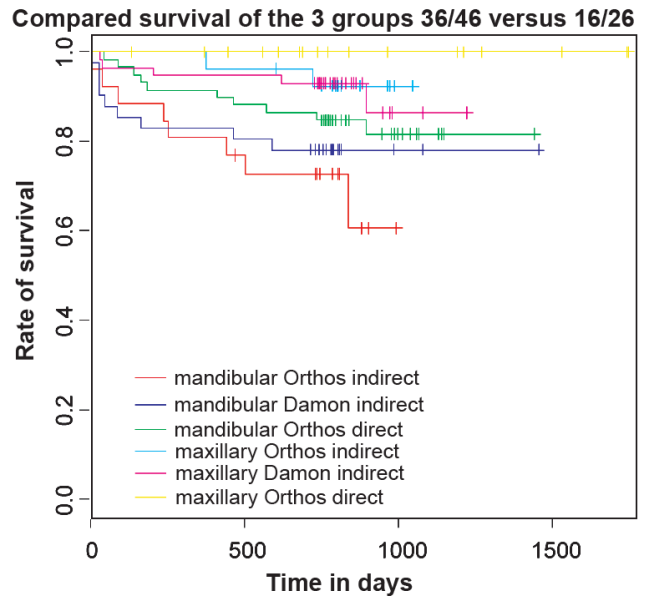

Figure 4

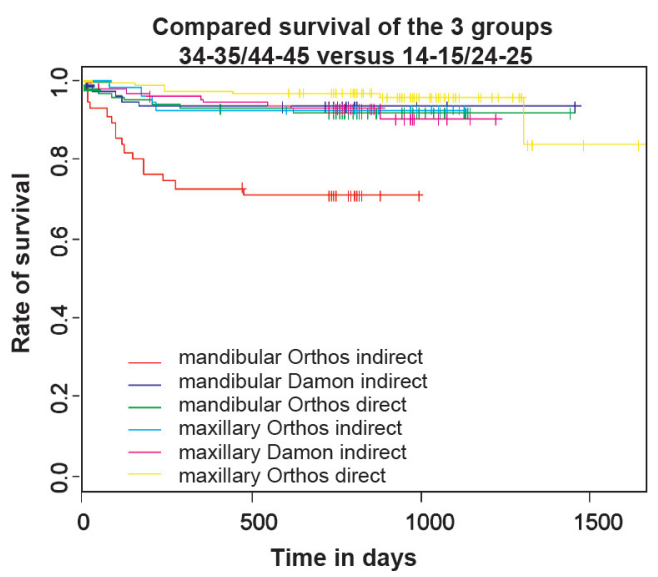

Figure 5

after 6 months, .727 after a year, and .709 after two years.

Direct bonding of Orthos attachments on canines gave the best results of the three groups (fig. 6). However, indirect bonding with Damon attachments demonstrated a performance similar to that of direct bonding of Orthos attachments on maxillary teeth.

The incisor sector showed even better performance with direct bonding of Orthos attachments (fig. 7).

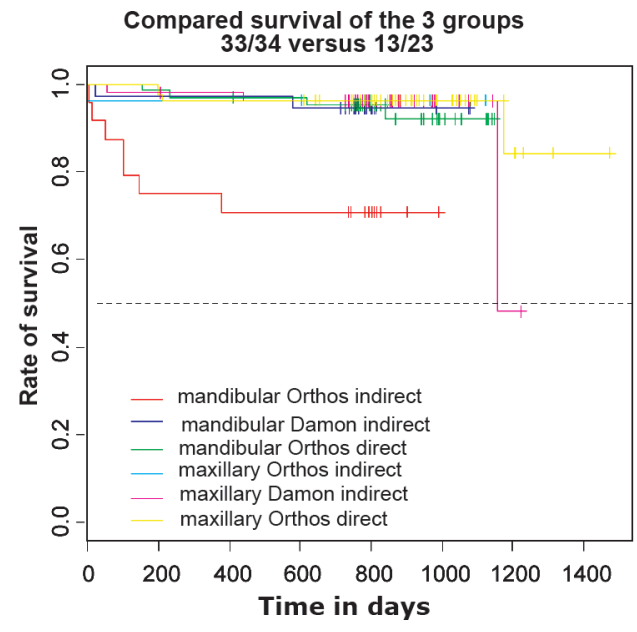

Figure 6

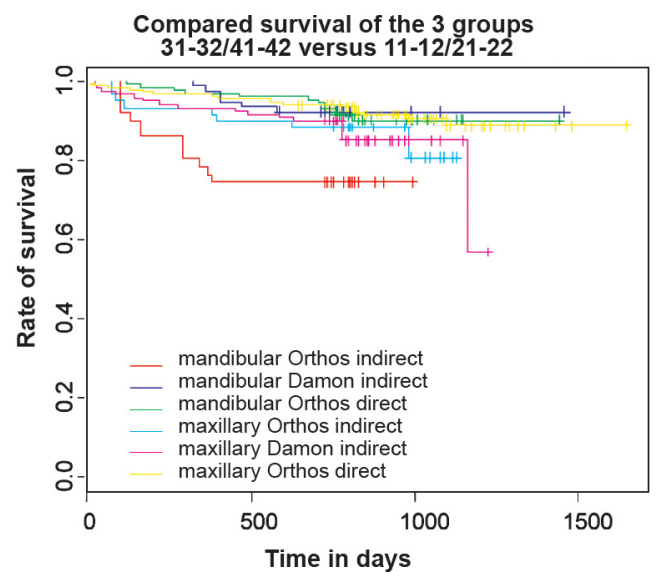

Figure 7

Indirect bonding with Damon attachments showed performance close to that of the direct Orthos incisor group. All of the attachments had better survival rates when they were bonded to mandibular incisors than they did with maxillary incisors except for the Orthos indirect group. 


\section{4 - DISCUSSION}

\section{4 - 1 - Discussion of the sample and of the criteria for inclusion}

The size of our sample was, it must be admitted, small and this may have masked inter-group differences. To assure this not happening we would have needed 350 subjects in each of the three groups.

Our sample was not homogenous in the sex of the subjects nor in the age of the patients in the different age classes (according to $\chi^{2}$ of table 1 ).

There was also a difference between the number of attachments bonded on the molars, on the premolars, on the canines, and on the incisors in the three groups of attachments Orthos Direct, Orthos indirect, and Damon indirect (according the $\chi^{2}$ of table 2).

We did not utilize the sample of Damon attachments bonded by the principal practitioner because there were not enough of them. We would have had to analyze a sample furnished by another practitioner, a procedure that might have introduced a bias into our study. For the same reason we did not utilize a control group with attachments bonded with composite because our principal practitioner doesn't use this material to bond to enamel. So it was the direct Orthos bondings that we selected as a control group.

We do not believe that missed appointments and an orthodontic-surgical protocol by themselves alone explain the low rate of survival of the indirectly bonded Orthos group.

\section{4 - 1 - 1 - Length of observation}

Our observation period coincided with the usual length of orthodontic treatment, which, on the average, is two years.

Because of this long time frame the bonding agents under study were frequently subjected to intraoral temperature changes greater than the point of vitreous transition, 54\%, which weakened bond strength provoking a higher rate of detachment ${ }^{2}$.

\section{4 - 1 - 2 - Characteristics of the attachments}

On the average, the surface area of the Orthos attachments is greater than that of the Damon attachments. Still, that greater surface area surprisingly confers no advantage because in indirect bonding their survival rate is lower than Damon's.

The Orthos attachments are thinner than the Damon attachments but this slimness doesn't appear to exert a positive effect on survival rate because the Damon group of attachments bonded indirectly to molars had a better survival rate than the Orthos indirect bonding group. In addition, in the indirect technique it is necessary to increase the thickness of the supplementary composite Transbond XT wedge even though in theory it is supposed to be as thin as possible.

The bases of the Orthos attachments are more rounded than those of the Damon attachments and their fit with the bicuspid surfaces seems better than that of the Damon group but this feature has no effect on the success of indirect bonding because the adaption to the teeth is accomplished by the intervening composite wedge.

The underside of the two types of brackets consists of an Optimesh treatment grid, 80 gauge for the 
Damon attachments and 100 gauge for the Orthos. So one would expect a poorer adhesion between Fuji Ortho LC and the Orthos base in the direct technique. In the same way, one would expect a more retentive bond of the base of the Damon attachment with composite, contentions our results confirmed.

In this study we did not prepare any of the attachment brackets chemically or mechanically.

\section{4 - 2 - Discussion of the protocols}

It is possible that the composite wedge may remain polluted even though it has been treated with acetone because this application merely removes fats and salivary glycoproteins. However it is not smoothed out and no composite adhesive material is applied to it.

Based on his clinical experience, G. Altounian advises bonding to dry enamel not covered with particles of water during indirect lingual bonding because it is impossible to exert right angle pressure on each attachment during the process ${ }^{1}$. However, in our study we bonded all the attachments to a slightly moist enamel surface.

In direct bonding, photopolymerization is accomplished in a single stage. But in indirect bonding two stages, separated by a 5 minute interval, are used to complete photopolymerization, which increases the possibility of contamination, especially from saliva.

Orthodontists frequently do a full mouth bonding in two stages. First the transfer splints are prepared sepa- rately and checked in the mouth. If interference high spots are found, the operator can grind them away. In this technique the composite wedges can be placed the day of bonding or the week before.

In this case, wedges of glass ionomer cement are positioned the same day or the week before the bonding. In 2002, D.J. Hegarty showed that attachments bonded in an overbite situation are four times more likely to become detached than those placed where no overbite is present ${ }^{3}$.

The orthodontist ties the first arch in place 5 to $10 \mathrm{~min}$ after the final polymerization. This delay should be slightly longer for indirect bonding procedures.

A delay slightly more important for indirect bonding. In the literature, authors suggest waiting for one half hour to an hour before arches are ligated in place, a delay that we and others did not respect ${ }^{5,6}$. This may account for the relatively low rate of bonding survival we registered.

The Orthos attachments are secured with metallic or elastomeric ligatures depending upon the stage of treatment. Opening the automatic housing of the first generation selfligating Damon brackets with the special pliers proposed for that use by the manufacturer did not seem to have any effect on survival rate.

The pressure exerted on Resin Modified Glass- Ionomer Cement bond joints by Class II intermaxillary elastics or normal intercuspation in our sample did not seem to affect maxillary canines before the 1200 day mark. 


\section{4 - 3 - Clinical performance of RMGIC}

\section{4 - 3 - 1 - Comparison of our rates of detachment and rates of survival with those reported in the litera- ture for direct bonding with RMGIC}

Our overall rate of detachment of directly bonded brackets, $14.5 \%$, is higher than rates reported in the literature. But the rate of detachment of the Orthos Direct group, $8.5 \%$, is lower than rate D. J. Hegarty reported in his 2002 paper $^{3}$. And our detachments occurred later in treatment.

Our survival rate for directly bonded brackets is .963 at 8 months, .952 at one year, .943 at 1.2 years, and .936 at 18 months. This is lower than the survival rate of .96 after a year that $A$. Summers reported in $2004^{\circ}$ but he used brackets whose base area was smaller that ours. It is higher than $\mathrm{E}$. Silverman's rate, in 1995, of 96.8 at 8 months $^{8}$, of I. Shammaa's rate, in 1996, of .86 at 1.2 years $^{7}$, and of D. J. Hegarty's rate, in 2002, of .90 at one year $^{3}$. It is also higher than L. Hitmi's rate, in 2001, of .918 at 8 months ${ }^{4}$. This might be explained by the hetero- geneity of Hitmi's sample ${ }^{4}$, which included three types of brackets, metal, ceramic, and plastic.

Our rate of survival of attachments to first molars was 0.885 at 18 months. Our rate of survival of attachments to second molars was 0.797 at 18 months. The average rate is almost the same as the rate found in the study Hitmi conducted on molars in $2001^{4}$.

\section{4 - 3 - 2 - Comparison of our detachment rate and our survival rate with data in the literature on direct bonding with RMGIC}

The rate of survival of the attachments we bonded indirectly was .927 at 6 months, .918 at 8 months, .906 at one year, .90 at 14 months, and .891 at 18 months. These figures are all a little lower than the rates reported in the literature for the survival of directly bonded attachments.

We were unable to find any articles dealing with indirect bonding with RMGIC. All the authors of the articles we reviewed performed bonding procedures with composite materials

\begin{tabular}{|c|c|c|c|c|c|c|c|c|c|c|c|}
\hline & & \multicolumn{2}{|c|}{ Incisors } & \multicolumn{2}{|c|}{ Canines } & \multicolumn{2}{|c|}{ Premolars } & \multicolumn{2}{|c|}{ First molars } & \multicolumn{2}{|c|}{ Second molars } \\
\hline & & Sup. & Inf. & Sup. & Inf. & Sup. & Inf. & Sup. & Inf. & Sup. & Inf. \\
\hline \multirow{3}{*}{$\begin{array}{l}\text { Orthos } \\
\text { Direct }\end{array}$} & 6 months & 0.975 & 0.985 & 1 & 0.985 & 0.987 & 0.948 & 1 & 0.932 & 0.923 & 0.955 \\
\hline & 12 months & 0.97 & 0.969 & 1 & 0.97 & 0.974 & 0.928 & 1 & 0.915 & 0.923 & 0.909 \\
\hline & 24 months & 0.94 & 0.931 & 0.963 & 0.954 & 0.967 & 0.917 & 1 & 0.864 & 0.923 & 0.816 \\
\hline \multirow{3}{*}{$\begin{array}{l}\text { Orthos } \\
\text { Indirect }\end{array}$} & 6 months & 0.933 & 0.863 & 0.963 & 0.75 & 0.962 & 0.764 & 0.963 & 0.885 & 0.857 & 0.619 \\
\hline & 12 months & 0.933 & 0.784 & 0.963 & 0.708 & 0.925 & 0.724 & 0.963 & 0.808 & 0.857 & 0.524 \\
\hline & 24 months & 0.883 & 0.745 & 0.963 & 0.708 & 0.925 & 0.709 & 0.923 & 0.726 & 0.821 & 0.429 \\
\hline \multirow{3}{*}{$\begin{array}{l}\text { Damon } \\
\text { Indirect }\end{array}$} & 6 months & 0.95 & 1 & 0.981 & 0.973 & 0.969 & 0.932 & 0.966 & 0.829 & 0.914 & 0.889 \\
\hline & 12 months & 0.933 & 0.987 & 0.981 & 0.973 & 0.953 & 0.932 & 0.948 & 0.829 & 0.857 & 0.889 \\
\hline & 24 months & 0.9 & 0.922 & 0.962 & 0.946 & 0.929 & 0.93 & 0.93 & 0.78 & 0.829 & 0.815 \\
\hline
\end{tabular}

Table 3

The survival rates at 6, 12, and 24 months. 
that were chemopolyerized. Although direct bonding with composites and indirect bonding with composites yield similar results, direct bonding with Fuji Ortho LC works better than indirect bonding with that material. Under the conditions and in accor- dance with the protocols of our study, it would seem that this material adheres better to enamel than does the composite wedge and that the multiplicity of materials and of interfaces weakens bond strength in the indirect technique.

\section{5 - CONCLUSION}

Our study makes it clear that direct or indirect bonding with Fuji Ortho LC works effectively in the private daily practice of orthodontics. Our accidental bracket detachment rate and our survival rates are acceptable except for Orthos attachments bonded indirectly to mandibular teeth.

Under the conditions prevailing in our study direct bonding worked slightly better than indirect bonding. In the Damon Indirect and the Orthos Direct groups the survival rate of the attachments on the second molars was worse than the rates on all the other teeth.
Practitioners should gain a better understanding of some of the parameters influencing bonding with resin modified glass-ionomer cement in order to improve their bonding skills, especially how to choose the attachment that will work best with a specific bonding material and how to deal with the effect of overbite on the stability of bonded junctions. It should be noted that the multiplicity of interfaces and materials that were part of the framework of our applications of the indirect technique weakened the bond strengths achieved under the conditions of and in accordance with the protocols of our study.

\section{BIBLIOGRAPHY}

1. Altounian G. Le collage orthodontique aux verre-ionomères: de la théorie à la pratique. Rev Orthop Dento Fac 2000;34:557-99.

2. Azevedo C., Forestier J.P., Tavernier B. Effect of Time on the Flexural Strength of Gloass IO,omer and Composite Orthodontic Adhesives. Angle Orthod 2004;75:114-8.

3. Hegarty D.J., Macfarlane T.V. In vivo bracket retention comparison of a resin-modified glass ionomer cement and a resin-based bracket adhesive system after a year. Am J Orthod Dentofacial Orthop 2002;121:496-501.

4. Hitmi L., Muller C., Mujajic M., Attal J.-P. An 18-month clinical study of bond failures with resin-modified glass ionomer cement in orthodontic practice. Am J Orthod Dentofacial Orthop 2001;120:406-15.

5. Janier S. L'effet du temps, du timing et de la nature de l'énergie photonique sur le comportement physico-mécanique du Fuji Ortho LC. Thèse d'exercice de Chirurgie Dentaire, 2007, Paris VII.

6. Palucha A. Evaluation de l'influence du traitement de surface à l'intrados des grilles des attaches orthodontiques sur l'adhérence de l'interface : grille / ciment verre ionomère modifié par adjonction de résine. Mémoire pour le CECSMO, 2005, Paris VII. 
7. Shammaa I., Ngan P., Kim H., Kao E., Gladwin M., Gunel E., Brown C. Comparison of bracket debonding force between two conventional resin adhesives and a resin-reinforced glass ionomer cement: An in vitro and in vivo study. Angle Orthod 1999;69(5):463-9.

8. Silverman E., Cohen M., Demke R.S., Silverman M. A new light-cured glass ionomer cement that bonds brackets to teeth without etching in presence of saliva. Am J Orthod Dentofac Orthop 1995; 108:231-6.

9. Summers A., Kao E., Gilmore J., Gunel E., Ngan P. Comparison of bond strength between a conventional resin adhesive and a resin-modified glass ionomer adhesive: An in vitro and in vivo study. Am J Orthod Dentofacial Orthop 2004;126:200-6. 